\title{
Metabolomics analysis reveals Embden Meyerhof Parnas pathway activation and flavonoids accumulation during dormancy transition in tree peony
}

Tao Zhang ${ }^{1,2}$, Yanchao Yuan ${ }^{1,2}$, Yu Zhan ${ }^{1,2}$, Xinzhe Cao ${ }^{1,2}$, Chunying Liu ${ }^{1,2}$, Yuxi Zhang ${ }^{1,2^{*}}$ and Shupeng Gai ${ }^{12^{*}}$

\begin{abstract}
Background: Bud dormancy is a sophisticated strategy which plants evolve to survive in tough environments. Endodormancy is a key obstacle for anti-season culture of tree peony, and sufficient chilling exposure is an effective method to promote dormancy release in perennial plants including tree peony. However, the mechanism of dormancy release is still poorly understood, and there are few systematic studies on the metabolomics during chilling induced dormancy transition.

Results: The tree peony buds were treated with artificial chilling, and the metabolmics analysis was employed at five time points after $0-4{ }^{\circ} \mathrm{C}$ treatment for $0,7,14,21$ and $28 \mathrm{~d}$, respectively. A total of 535 metabolites were obtained and devided into 11 groups including flavonoids, amino acid and its derivatives, lipids, organic acids and its derivates, nucleotide and its derivates, alkaloids, hydroxycinnamoyl derivatives, carbohydrates and alcohols, phytohormones, coumarins and vitamins. Totally, 118 differential metabolites $(V I P \geq 1, P<0.05)$ during chilling treatment process were detected, and their KEGG pathways involved in several metabolic pathways related to dormancy. Sucrose was the most abundant carbohydrate in peony bud. Starch was degradation and Embden Meyerhof Parnas (EMP) activity were increased during the dormancy release process, according to the variations of sugar contents, related enzyme activities and key genes expression. Flavonoids synthesis and accumulation were also promoted by prolonged chilling. Moreover, the variations of phytohormones (salicylic acid, jasmonic acid, abscisic acid, and indole-3-acetic acid) indicated they played different roles in dormancy transition.
\end{abstract}

Conclusion: Our study suggested that starch degradation, EMP activation, and flavonoids accumulation were crucial and associated with bud dormancy transition in tree peony.

Keywords: Tree peony, Dormancy transition, Metabolomics, EMP activation, Flavonoids accumulation

\footnotetext{
*Correspondence: zhang-yuxi@163.com; spgai@qau.edu.cn

'College of Life Sciences, Qingdao Agricultural University, Qingdao 266109,

China

Full list of author information is available at the end of the article
}

(c) The Author(s). 2020 Open Access This article is licensed under a Creative Commons Attribution 4.0 International License, which permits use, sharing, adaptation, distribution and reproduction in any medium or format, as long as you give appropriate credit to the original author(s) and the source, provide a link to the Creative Commons licence, and indicate if changes were made. The images or other third party material in this article are included in the article's Creative Commons licence, unless indicated otherwise in a credit line to the material. If material is not included in the article's Creative Commons licence and your intended use is not permitted by statutory regulation or exceeds the permitted use, you will need to obtain permission directly from the copyright holder. To view a copy of this licence, visit http://creativecommons.org/licenses/by/4.0/ The Creative Commons Public Domain Dedication waiver (http://creativecommons.org/publicdomain/zero/1.0/) applies to the data made available in this article, unless otherwise stated in a credit line to the data. 


\section{Background}

The bud dormancy of woody plants is a complex process that allows plants to survive in harsh environments such as cold and drought, and it is classified as paradormancy, endodormancy, and ecodormancy [1], of which endodormancy is regulated by internal factors [2]. Endodormancy also has an important impact on the maintenence and producction of the plant, for instance, the anti-season cultivation of some fruit trees. Thus, much attention has been attracted to understand the mechanism of dormancy regulation. External environmental factors, short-day and low temperature, play an essential role in endodormancy induction, and sufficient low temperature accumulation is a necessary prerequisite to bud break [3-5]. In Paeonia lactiflora, low temperature accumulation at $5{ }^{\circ} \mathrm{C}$ for nine weeks was required to ensure the re-growth of buds [6]. Also, the buds of tree peony 'Luhehong' need to undergo $21 \mathrm{~d}$ chilling at $0-4{ }^{\circ} \mathrm{C}$ to ensure the following normal development at growth condition [7]. In addition, the requirements of chilling unit are in a genotype dependent manner [8], and significantly different among apical buds, lateral buds and catkins in Persian walnut (Juglans regia L.) [9].

Many studies have been performed on the mechanism of bud dormancy release, such as genomics, transcriptomics, proteomics and molecular biological analysis. These results indicated that vast changes in the metabolism including carbohydrate inter-conversion and transport, lipid mobilization, nitrogen metabolism, phytohormone metabolism and redox processes are associated with chilling induced buds dormancy break $[8$, 10-13]. Till now, there is still rare study on the systematic changes of metabolites and their crosslinks during chilling induced dodormancy transition.

Carbohydrates play multiple roles in plant growth and development, besides acting as the primary source of carbon and energy. Before oxidative phosphorylation, Embden Meyerhof Parnas (EMP), Tricarboxylic Acid (TCA), and Pentose Phosphate Pathway (PPP) are the main respiration pathways in plants. EMP starts from glucose, which is an end product of starch degradation. Additionally, maltose and fructose also involve in the EMP pathway after conversion to glucose. The anabolic metabolism of sucrose is mainly carried out by two enzymes, sucrose synthase (SUS; EC 2.4.1.13) and invertase (INV; EC3.2.1.26) [14]. SUS reversibly catalyzes the formation of sucrose from UDP-glucose and fructose [15], and INV, which irreversibly decompose sucroses into hexose, can be divided into three categories: cell-wall invertase (CWIN), vacuolar invertase (VIN), and cytoplasmic invertase (CIN) $[16,17]$.

It had been reported that EMP, TCA, and PPP are strictly related to dormancy release in different plants.
For example, the TCA cycle is enhanced, while the PPP pathway slowly decreased during apple bud sprouting [18]. In grape, dormancy release induced by chemical and low temperature was found related to PPP, EMP, and TCA cycles [19-21]. Furthermore, carbohydrates could also act as a sugar signaling molecule. Mason et al. (2014) found that sucrose could serve as a signaling molecule involved in paradormancy release [22].

Flavonoids are widespread secondary metabolites in plants, which mainly contain six subclasses: chalcones, flavones, flavonols, flavandiols, anthocyanins, and proanthocyanidins or condensed tannins [23]. The pathway of flavonoid biosynthesis is quite conservative and well understood in some model plants [23]. Some genes involved in the production of common precursors, such as chalcone synthase $(\mathrm{CHS})$, chalcone isomerase $(\mathrm{CHI})$, flavanone 3-hydroxylase (F3H), and flavonoid 3'-hydroxylase $\left(F 3^{\prime} H\right)$, are called Early Biosynthetic genes (EBGs). Correspondingly, downstream genes for flavonoid biosynthesis are called Late Biosynthetic genes (LBGs). The pathway of flavonoids biosynthesis can be affected by biotic and abiotic factors (e.g., pathogen infections, temperature, drought, plant hormones) [24]. Moreover, recent reports had shown that flavonoids involved in plant stress response [25], pollen development [26], color formation [27], etc. However, it is still unknown whether flavonoids participate in the process of dormancy release.

It is well known that external factors always work through internal factors during bud dormancy release, and hormones play an important role in dormancy regulation. With the extension exposure to dormancyinducing conditions (short-day or low temperature), the expression of growth-promoting signals gene (FLOWERING LOCUS T, FT) is inhibited, leading to reduce gibberellins (GAs) levels and increase abscisic acid (ABA) contents, and the $\mathrm{ABA}$ response could induce the close of plasmodesmata, thereby mediating the establishment of dormancy $[28,29]$. GA and ABA not only involve in the establishment of growth cessation, but also play a key role in dormancy release. During dormancy release, the reopening of plasmodesmata could restore the supply of growth-promoting signals with the increasing biosynthesis of GAs [30]. On the other hand, the degradation of ABA is necessary for bud dormancy release in grapes, while ABA is accumulated during dormancy establishment [31]. The recent reports showed that SHORT VEGETATIVE PHASE (SVP)-like (SVL) with sequence homology to the Dormancy Associated MADS-box (DAM) genes [32], plays a vital role in the dormancy of poplar [33]. Low-temperature decreases ABA levels and reduces $S V L$ expression, leading to the induction of FT1 expression and GA biosynthesis, which promotes dormancy release. 
Tree peony (Paeonia suffruticosa Andr.) belonging to the Moutan subfamily of the genus Paeonia, Paeoniaceae, is one of the most ancient ornamental and medicinal plants in the world. The bud of tree peony is a typical compound bud with scale forming in autumn [34], and it must undergo a period of low temperature to ensure the sprouting and flowering in the next year. Due to the short and concentrated florescence every year, its anti-season culture becomes an important content of the tree peony industry. Until now, the primary method of anti-season production is to provide sufficient low temperature exposure alone or chilling enduration combining with gibberellin application. Therefore, it is of great value to understand the mechanism of chilling induced dormancy release in tree peony. Our previous study characterized the relationship between chilling accumulation and dormancy status, the physiological status of tree peony 'Luhehong' after $14 \mathrm{~d}$ chilling treatment was regarded as the transition stage from endodormancy to endodormancy release, and that after $21 \mathrm{~d}$ chilling treatment was defined as dormancy release, and after $28 \mathrm{~d}$ chilling as a stage of ecodormancy [35]. GA pathway plays a crucial role in endodormancy release induced by chilling [36]. And the activity of PPP pathway also increases during the process, suggesting that it play a role in dormancy release of tree peony [7]. As known, traits are more closely related to metabolites, which may provide a new perspective for the understanding of dormancy transition in tree peony.

Here, metabolic changes of tree peony buds during the chilling induced dormancy transition were analyzed. Kyoto Encyclopedia of Genes and Genomes (KEGG) enrichment analysis showed that differential metabolites involved in various metabolic pathways such as carbon metabolism, secondary metabolite synthesis, and hormone metabolism. It was revealed that starch degradation and EMP activity were enhanced during dormancy release. Interestingly, flavonoids anabolism was also activated by chilling accumulation, and its increasement might in return promote flower bud development. Furthermore, the concentration of plant hormones, such as ABA, jasmonic acid (JA), salicylic acid (SA), and indole3 -acetic acid (IAA) during the dormancy transition were also evaluated in this research. Significantly, the roles of flavonoids were firstly discussed during the dormancy transition in perennial plants. All results would provide valuable information for the molecular mechanism of dormancy transition in tree peony.

\section{Results}

Metabolomics analysis during chilling induced dormancy transition

To study the metabolic changes during chilling-induced dormancy release in tree peony, flower buds were picked at five time points after $0-4{ }^{\circ} \mathrm{C}$ treatment for $0,7,14,21$ and $28 \mathrm{~d}$, respectively, and metabolic profiling was analyzed by ultra-performance liquid chromatography (UPLC) and tandem mass spectrometry (MS/MS) (Fig. 1a). The Principal Component Analysis (PCA) analysis was employed to evaluate the repeatability of the metabolite profiles (Fig. 1b). As shown, quality control (QC) samples were separated from tested samples, and the two principal components (PC) accounted for $28.8 \%$ (PC1) and 14.5\% (PC2) of the total variance, respectively. The samples were clearly separated into the 5 different subgroups by PC1 and PC2 (Fig. 1b).

A total of 535 metabolites were detected in the metabolomic analysis, and 511 of them were annotated with MassBank, KNAPSAcK, HMDB [37], MoTo DB and METLIN [38] (Table S1). The metabolites were divided into eleven groups, including flavonoids, amino acid and its derivatives, lipids, organic acids and its derivates, nucleotide and its derivates, alkaloids, hydroxycinnamoyl derivatives, carbohydrates and alcohols, phytohormones, coumarins, and vitamins (Table 1). Notebly, 123 flavonoids and derivates and 15 phytohormones were annotated (Table S1), which benifited to analyze their function. The results of metabolic pathway analysis showed that they were mapped to 14 KEGG pathways (Fig. 1c), of them, totally 183 metabolites were assigned to the pathway of global and overview, followed by amino acid metabolism with 85 , biosynthesis of other secondary metabolites numbered 82 , metabolism of cofactors and vitamins numbered 33, nucleotide metabolism, carbohydrate metabolism amounted 33 , and so on (Fig. 1c).

\section{Differential metabolites analysis}

The Orthogonal Projection to Latent StructuresDiscriminant Analysis (OPLS-DA, VIP $\geq 1$ ) and the Student's $\mathrm{t}$ test $(P<0.05)$ were applied to detect the differential metabolites (DMs) among different treatments (Fig. S1). Totally, 118 DMs were obtained when the chilling treated groups were compared with that of $0 \mathrm{~d}$ samples. The results also indicated that the amounts of up-regulated metabolites increased along with the increase of chilling days (Fig. 2a). In detail, the number of up-regulated metabolites were 4, 18, and 29 when $14 \mathrm{~d}$ vs $0 \mathrm{~d}, 21 \mathrm{~d}$ vs $0 \mathrm{~d}$, and $28 \mathrm{~d}$ vs $0 \mathrm{~d}$, respectively. There were not regular trend for the number of downregulated metabolites when compared with $0 \mathrm{~d}$. The results indicated that some metabolism pathways were activated by the prolonged chilling exposure. Interestingly, the number of up-regulated metabolites decreased when comparing $14 \mathrm{~d}$ vs $0 \mathrm{~d}$ with $7 \mathrm{~d}$ vs $0 \mathrm{~d}$. To better understand the metabolites changes caused by different chilling durations, the venn diagram of 118 DMs was constructed, showing that four metabolites were 


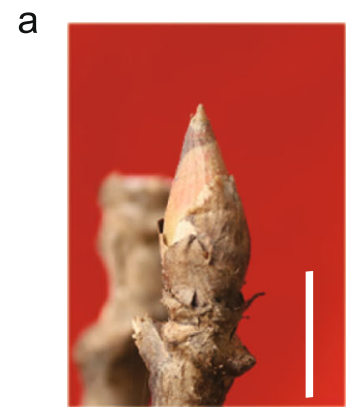

$0 d$

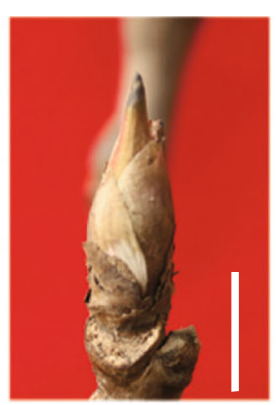

$7 d$

b

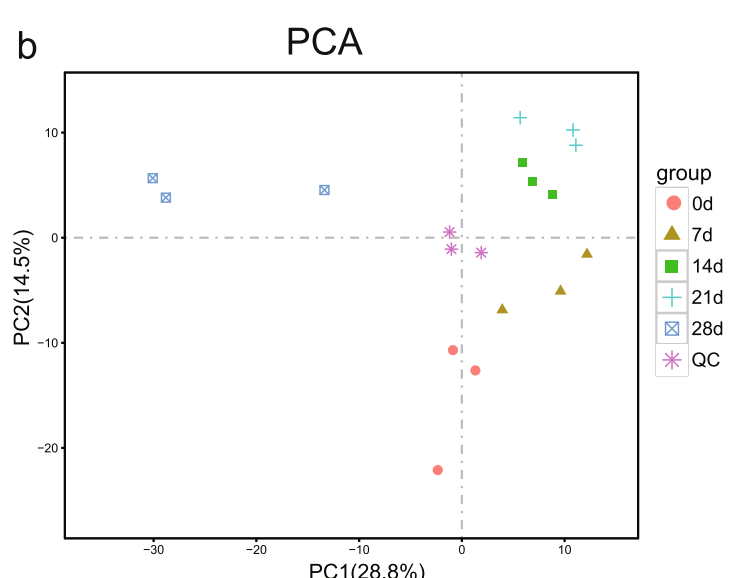

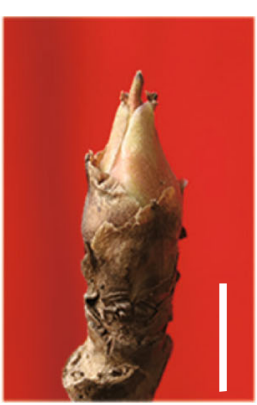

$14 \mathrm{~d}$

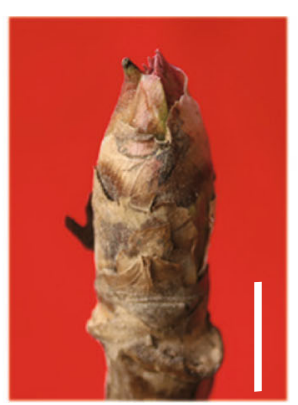

$21 d$

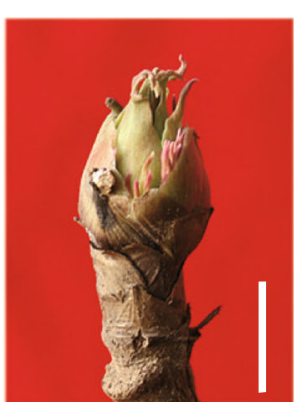

$28 d$

C

KEGG pathway annotation

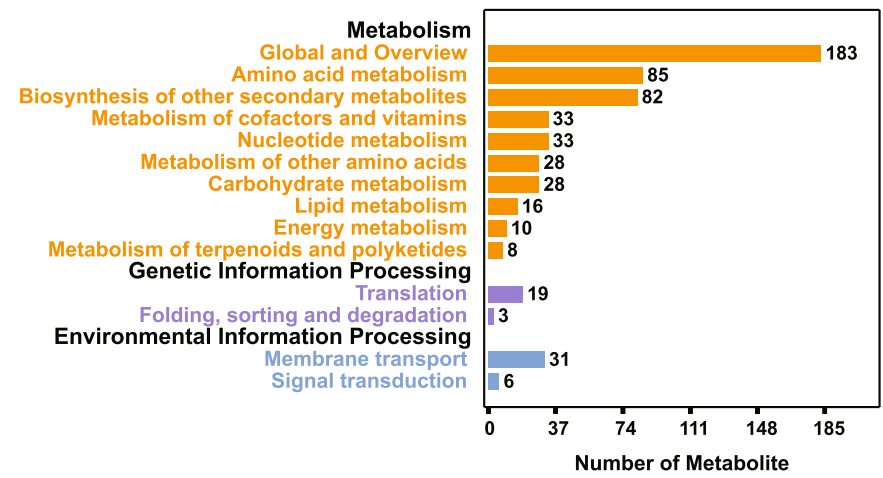

Fig. 1 Metabolomics analysis of tree peony buds. a Bud morphology of tree peony buds during the chilling duration. The scale bar indicated 1 $\mathrm{cm}$. b PCA plot of the tree peony buds at five stages. $0 \mathrm{~d}, 7 \mathrm{~d}, 14 \mathrm{~d}, 21 \mathrm{~d}$ and $28 \mathrm{~d}$ indicated days for chilling duration. QC, quality control samples. c The KEGG pathway annotation of metabolites. Black texts indicated the first hierarchy of KEGG Pathway database, and colored texts indicated the second hierarchy of KEGG Pathway database. The global and overview is a secondary metabolism name, containing eight metabolic pathways: Metabolic pathways, Biosynthesis of secondary metabolites, Microbial metabolism in diverse environments, Carbon metabolism, 2-Oxocarboxylic acid metabolism, Fatty acid metabolism, Biosynthesis of amino acids, Degradation of aromatic compounds

common among the four comparisons, i.e. $7 \mathrm{~d}$ vs $0 \mathrm{~d}, 14$ d vs 0 d, 21 d vs 0 d, and 28 d vs 0 d (Fig. 2b; Fig. S2). Besides, 30 metabolites were unique in $28 \mathrm{~d}$ vs $0 \mathrm{~d}$, implying that wider changes happened in this group. The KEGG pathway annotation of 118 DMs was performed and the DMs involved in amino acid metabolism (24 in

Table 1 Overview of annotated metabolites

\begin{tabular}{lll}
\hline Type & Number & Percentage (\%) \\
\hline Flavonoids & 132 & 25.83 \\
Amino acid and its derivatives & 79 & 15.46 \\
Lipids & 64 & 12.52 \\
Organic acids and its derivates & 62 & 12.13 \\
Nucleotide and its derivates & 51 & 9.98 \\
Alkaloids & 36 & 7.05 \\
Hydroxycinnamoyl derivatives & 27 & 5.28 \\
Carbohydrates and alcohols & 21 & 4.11 \\
Phytohormones & 14 & 2.74 \\
Coumarins & 9 & 1.76 \\
Vitamins & 9 & 1.76 \\
\hline
\end{tabular}

number), nucleotide metabolism (16), and biosynthesis of other secondary metabolism (15), and so on (Fig. 2c). Additionally, KEGG enrichments between each two treatments were listed in Fig. S3. Carbohydrate metabolisms, such as pyruvate metabolism, starch and sucrose metabolism, PPP pathway and so on, were frequently presented in the different enrichments analysis. Plant hormone signaling transduction was also enriched in 7 comparable groups. Besides above, several amino acid metabolisms, pyrimidine and purine metabolism, nitrogen metabolism and others were also frequently enriched in the comparations (Fig. S3).

\section{The metabolic processes related to dormancy release in tree peony}

To study the crucial metabolic processes related to endodormancy release in tree peony, DMs of $14 \mathrm{~d}$ vs $7 \mathrm{~d}$ and $21 \mathrm{~d}$ vs $7 \mathrm{~d}$ were screened. Totally, $50 \mathrm{DMs}$ were obtained and presented in a clustering heatmap, which showed the metabolites change from the dormancy to dormancy release (Fig. 3a). Among them, 21 DMs were significantly up-regulated, 18 DMs were down-regulated 


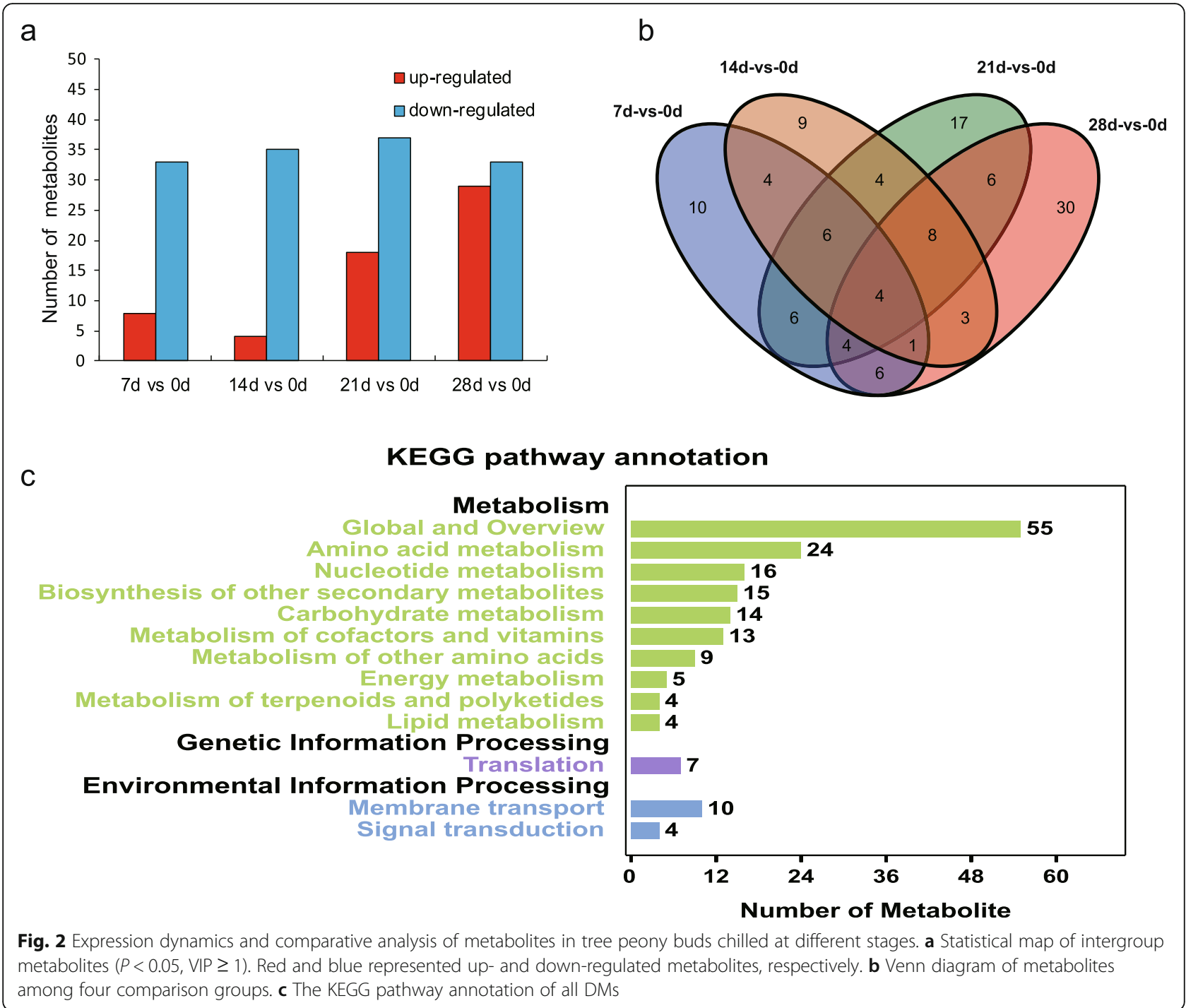

with the prolonged chilling enduration, and the other 11 DMs fluctuated with a peak at $14 \mathrm{~d}$. The KEGG analysis of the 50 DMs showed that 25 DMs participated in the metabolic pathway, which accounted for $83.33 \%$ of all the 30 annotated metabolites (Fig. 3b, Table S2). The KEGG enrichment anaylsis of 25 DMs showed that they involved in the pathways of glucose metabolism (glucose 6-phosphate), amino acid metabolism (aspartate) and hormone metabolism (ABA) (Table S3), suggesting that these metabolic pathways might play a critical role during dormancy release in tree peony.

\section{Carbon metabolism during dormancy transition in tree peony}

A metabolic network containing EMP pathway, TCA cycle, shikimate pathway, and amino acid metabolism was presented to visualize the carbon flow during dormancy release of tree peony (Fig. 4). The levels of glucose at 0,7 ,
14 and $21 \mathrm{~d}$ were lower than that at $28 \mathrm{~d}$. However, glucose 6-phosphate (G6P) and fructose 6-phosphate (F6P) had the higher levels at 7 and $14 \mathrm{~d}$ (Fig. 4), indicating that the EMP was activated during chilling induced endodormancy release in tree peony. In the TCA cycle, the levels of citrate declined until $14 \mathrm{~d}$ and then climbed. The succinic acid amount showed an significant upward at $14 \mathrm{~d}$. In terms of amino acid metabolism, some amino acids increased with the release of endodormancy, such as leucine, proline, etc., while some others decreased, such as valine, aspartic acid, glutamic acid, etc. (Fig. 4). In the shikimic acid pathway, the level of shikimic acid and phenylalanine increased after chilling exposure (Fig. 4).

\section{The variation of carbohydrates during dormancy transition}

Sugars play crutial roles in energy metabolism and substance metabolism, whose variation might reflect the 


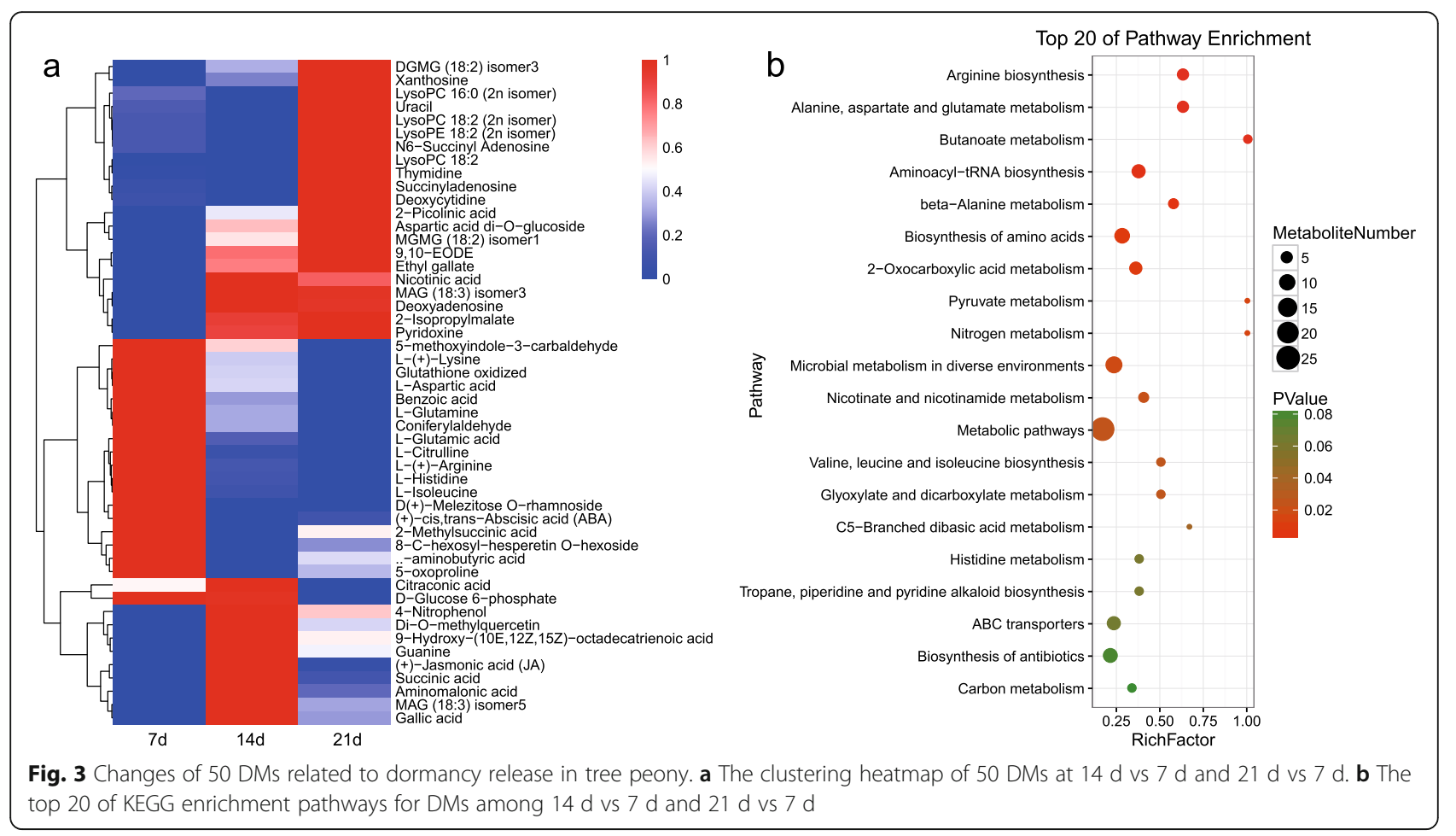

status of bud dormancy after chilling exposure. The levels of 15 sugars in metabolic profiles were analyzed throughout the dormancy process (Fig. 5a). Some monosaccharide (G6P, glucosamine, and trehalose 6phosphate) and polysaccharides (maltotetraose, melezitose, and melezitose $O$-rhamnoside) were up-regulated after $7 \mathrm{~d}$ chilling exposure, and then their contents reduced (Fig. 5a). The monosaccharides (fucose, glucose, and arabinose) had the maximum level at the ecodormancy stage ( $28 \mathrm{~d}$ chilling treated, Fig. 5a), indicating a well preparatory status for the following re-growth. A Gas Chromatography-Tandem Mass Spectrometry (GCMS/MS) measurement was then employed to further analyze the change of sugars during the chilling process in tree peony, and the results were similar to the metabolomics data. Fructose, glucose, and inositol were the three most abundant kinds of monosaccharides. The content of maltose significantly decreased after $7 \mathrm{~d}$ chilling treatment. Remarkably, the content of sucrose was the hightest of all the 13 tested sugars, which significantly increased and reached a maximum of $92.9 \mathrm{mg} / \mathrm{g}$ at $14 \mathrm{~d}$ chilling, and then declined rapidly (Fig. 5b), suggesting that it might play a vital role in the whole process. To further investigate the role of sucrose in dormancy release of buds, the expression patterns of sucrose synthase (PSSUS1 and PSSUS2) and sucrose invertase genes were analyzed (Fig. 5c, Fig. S4). The expression of PSSUS1 continued to decline after $7 \mathrm{~d}$ chilling treatment, but PSSUS2 was up-regulated at 7 and $14 \mathrm{~d}$ chilling. The expressions of cytoplasmic invertase (PSCIN), vacuolar invertase (PsVIN), and cell-wall invertase (PsCWIN) were significantly increased at different chilling periods (Fig. 5c). Taken together, it was presumed that sucrose catabolism was dominant during chilling duration process, to provide sufficient sugars for respiratory metabolism and energy metabolism. Starch, the main storage carbohydrate in higher plants, was measured during dormancy transition in tree peony. The results indicated that starch content decreased after chilling exposure and reached its minimum at $14 \mathrm{~d}$, which might be related to the activity of amylases (AMY) during the same process (Fig. 5d).

\section{The changes of flavonoids during dormancy transition} In KEGG enriched terms, the biosynthesis of secondary metabolites accounted for a large part (Fig. 3b), of which flavonoids are secondary metabolites that are widely present in plants. Thus a flavonoids metabolic pathway analysis was performed to understand its changes during dormancy transition in buds. Most flavonoids (naringenin, apigenin, luteolin, etc.), flavonols (kaempferol, quercetin, etc.), and anthocyanins (cyanidin 3-O-glucoside, pelargonidin 3-O-glucoside, etc.) showed similar down-regulated tendency at the early stages of dormancy $(0-14 \mathrm{~d})$ and up-regulated at $28 \mathrm{~d}$. Thus, their highest contents were usually detected at $28 \mathrm{~d}$, such as cyanidinbase anthocyanins, cyanidin 3-O-glucoside etc. (Fig. 6a). According to the results of LC-ESI-MS/MS, the relative 


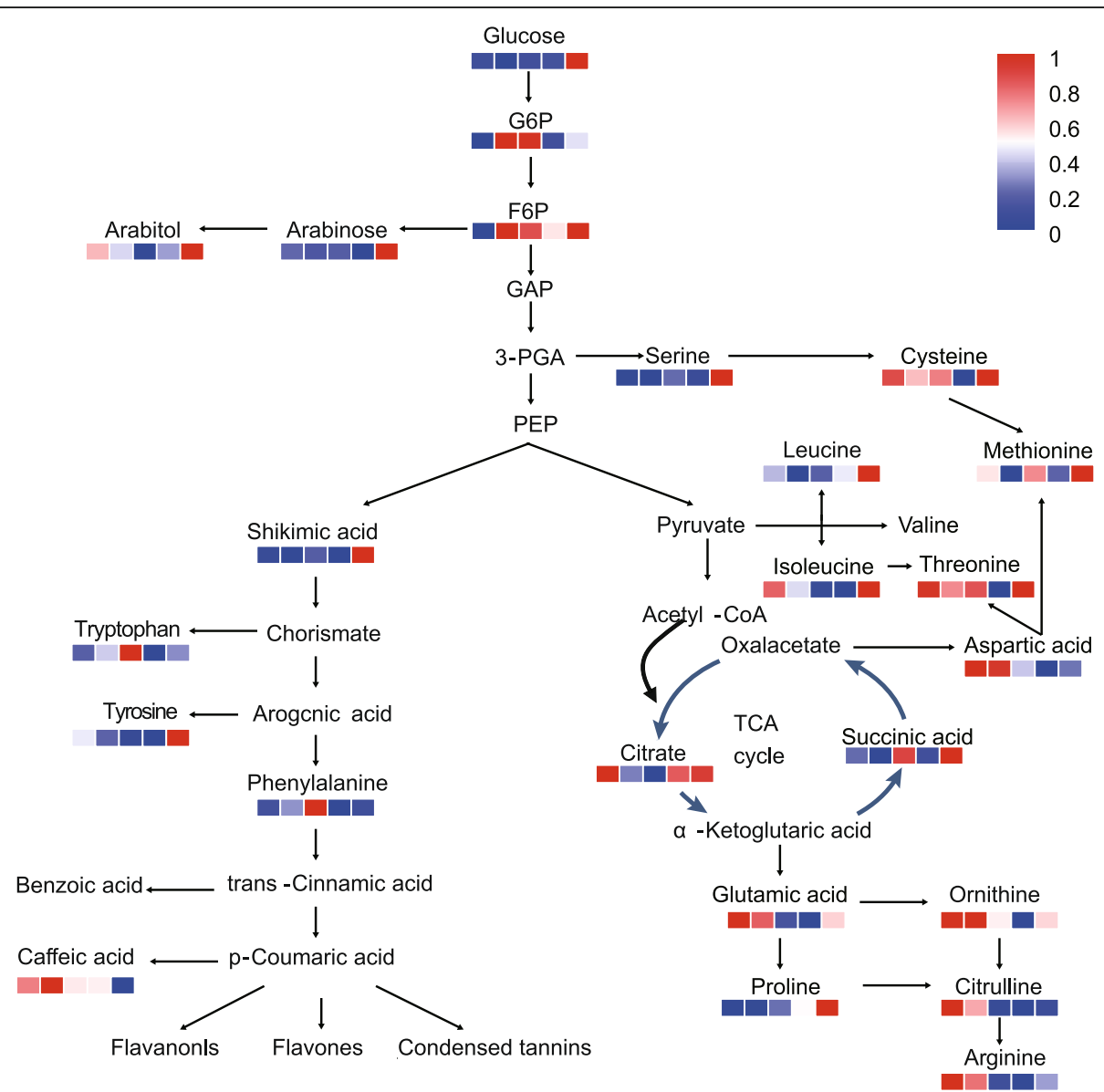

Fig. 4 Dynamics of carbon metabolic pathways throughout the chilling duration process. The metabolite amounts were shown in heatmaps as 0 , 7, 14, 21, and 28 d from left to right, respectively. G6P (glucose 6-phosphate), F6P (fructose 6-phosphate), GAP (3-phosphoglyceraldehyde), PEP (phosphoenolpyruvate)

contents of anthocyanins were analyzed at $28 \mathrm{~d}$. The results indicated that five anthocyanins, including cyanidin 3-O-glucoside (Cy3Glu), cyanidin 3-O-rutinoside (CyRut), cyanidin $\mathrm{O}$-syringic acid (CySyr), cyanidin 3,5di-O-glucoside (Cy3Glu5Glu), and pelargonidin 3-O-glucoside (Pg3Glu), were dominant in flower buds of tree peony, and the others were relactively rare (Fig. 6a and b). In detail, cyanidin-base anthocyanins (Cy3Glu5Glu) accounted for the majority, followed by Cy3Glu (Fig. 6a and b). Two EBGs (PsCHS and PsF3H) and two LBGs (PsANS and Ps3GT) showed the similar patterns excluding PSDFR. Their transcripts were relactively abundant at the beginning of dormancy $(0 \mathrm{~d})$, and dramatically declined at $7 \mathrm{~d}$ chilling, but prolonged chilling promoted their expression compared to $7 \mathrm{~d}$ treatment. The PsDFR remained very low expression level till dormancy release period, but significally increased by ten folds at $28 \mathrm{~d}$ comparing with $0 \mathrm{~d}$ (Fig. 6c, Fig. S4). The expression patterns of the raltaed genes were accordance with their content variations during chilling duration process.

\section{The variation of phytohormones during dormancy transition}

Phytohormones play vital roles in plant growth and development, flowering, stress response, and so on. In metabolomics analysis, 14 phytohormones or analogues, including SA, JA, GA, and ABA, were detected in dormant buds of tree peony, and their content variations during chilling duration were shown in a heatmap (Fig. 7a). Nine metabolites presented the highest contents at chilled $0 \mathrm{~d}$ (nonchilled period), and the other five hormones peaked at chilled $28 \mathrm{~d}$ (Fig. 7a). They were divided into three subgroups by cluster analysis The first subgroup showed a up-regulated tendency with a peak at $28 \mathrm{~d}$, including $\mathrm{SA}, \mathrm{GA}_{15}$, JA, methyl jasmonate (MeJA) and jasmonic acid-isoleucine (JA-Ile). Dihydrozeatin, salicylic acid $\mathrm{O}$-glucoside, trans-zeatin $\mathrm{N}$ glucoside and ABA were categorized into the second subgroup with an obviously down-regulated tendency. The others fluctuated during chilling exposure process with a peak at $0 \mathrm{~d}$ chilling point. 

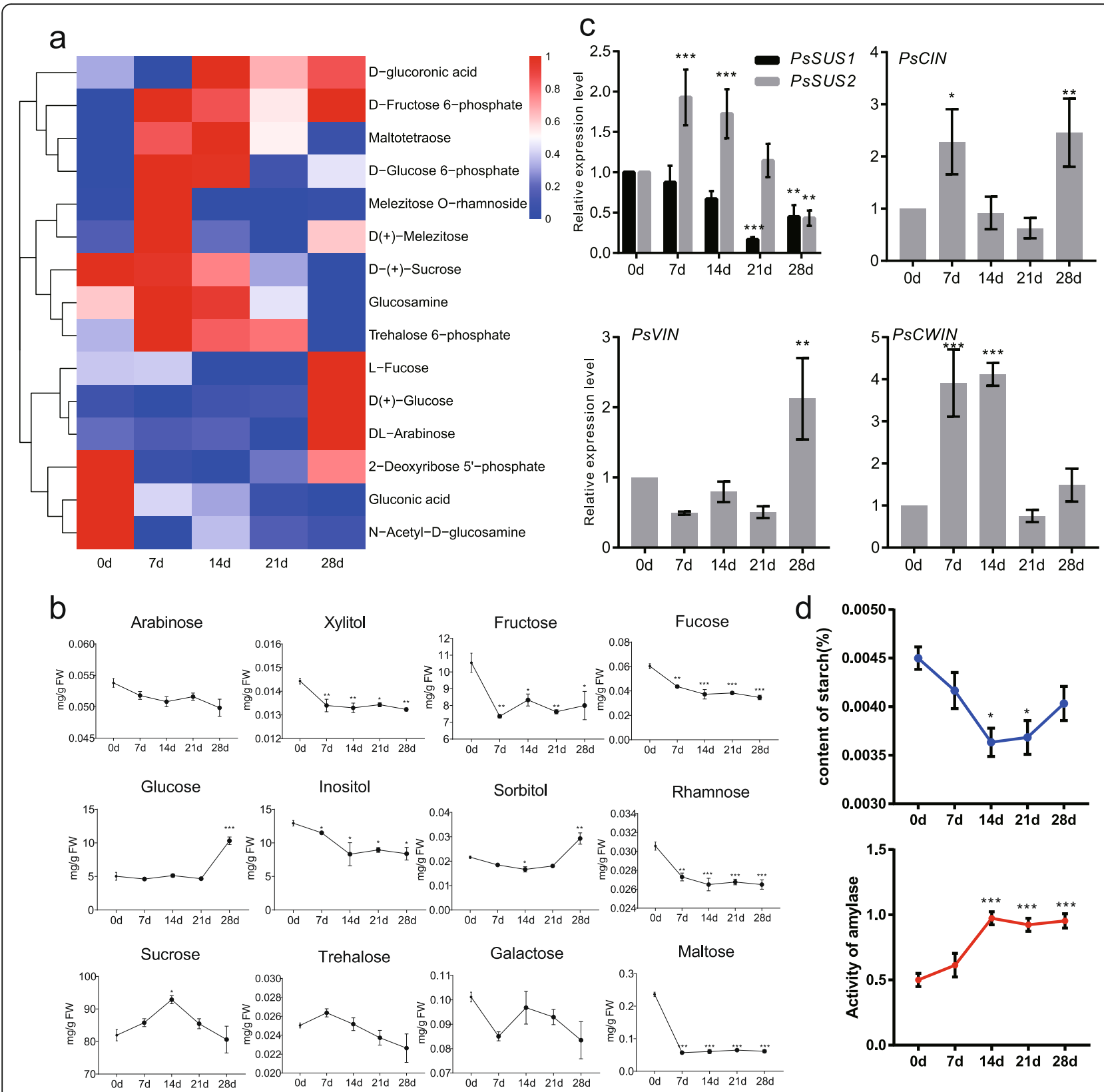

Fig. 5 Changes of carbohydrates during dormancy transition induced by the chilling in tree peony. a The cluster heatmap of carbohydrates. $\mathbf{b}$ The variations of several sugars content. $\mathbf{c}$ The relative expression levels of sucrose synthase and sucrose invertase genes. $\mathbf{d}$ The variations of starch and amylases activity. The mean \pm SD in three biological replicates was shown. * ${ }^{* *}$ and ${ }^{* *}$ indicated significant differences of one-way ANOVA at $P<0.05, P<0.01$, and $P<0.001$, respectively

The contents of JA, SA, IAA and ABA were also evaluated by LC-MS/MS analysis, and the results were similar to the metabolomics data. JA levels were relatively low from 0 to $21 \mathrm{~d}$ chilling, and dramatically peaked to $299.167 \mathrm{ng} / \mathrm{g}$ with a ten folds increase at chilled 28 d (Fig. 7b). The MYC2 (myelocytomatosis protein 2) transcription factor plays a central role in JA signal transduction [39]. Consistent with the variation of JA contents, the transcript of PsMYC2 increased sharply at chilled 28 d (Fig. 7c, Fig. S4). The contents of SA were downregulated from $0 \mathrm{~d}$ to $21 \mathrm{~d}$, and then recovered the initial content of $0 \mathrm{~d}$. The contents of IAA fluctuated during the chilling duration process, reached the highest level of $44.57 \mathrm{ng} / \mathrm{g}$ at $14 \mathrm{~d}$, and its content of $28 \mathrm{~d}$ was also higher than 0 and $21 \mathrm{~d}$ (Fig. $7 \mathrm{~b})$. The expression pattern of two auxin receptors genes (TRANSPORT INHIBITOR RESPONSE1/ 


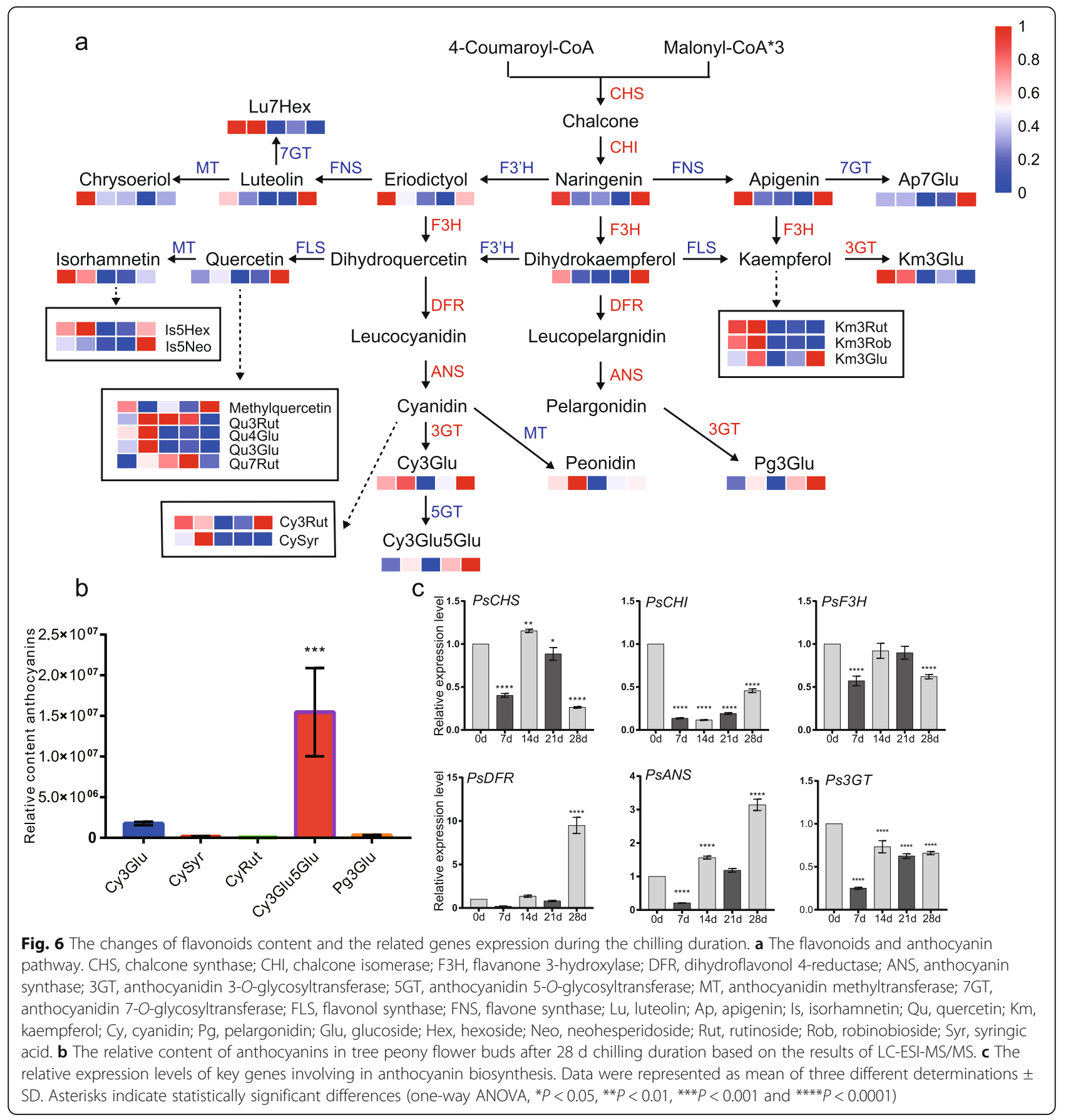

AUXIN SIGNALING F-BOX PROTEIN2,TIR1/AFB2) were analyzed during the chilling duration. PSAFB2 was down-regulated in the whole process, and PSTIR1 was up-regulated at $7 \mathrm{~d}$, and then downregulated from 7 to $28 \mathrm{~d}$ (Fig. 7c, Fig. S4). The contents of ABA persistently decreased from $1140.37 \mathrm{ng} /$ $\mathrm{g}$ at $0 \mathrm{~d}$ to $803.83 \mathrm{ng} / \mathrm{g}$ at $28 \mathrm{~d}$ (Fig. 7b), indicating that $\mathrm{ABA}$ was an inhibitor of dormancy release in tree peony buds, and chilling treatment could gradually reduce the contents of $\mathrm{ABA}$.

\section{Discussion}

The dormancy transition of woody plants is a complicated process, which was synergetically regulated by photoperiod and low temperature [40]. Chilling treatment as an effective method to promote dormancy release had been verified in many species including tree peony $[3-5,9,41]$. Transcriptomics, proteomics and microRNAs analysis had been performed to investigated the complex mechanism of chilling induced dormancy release in tree peony, which revealed the roles of GA 


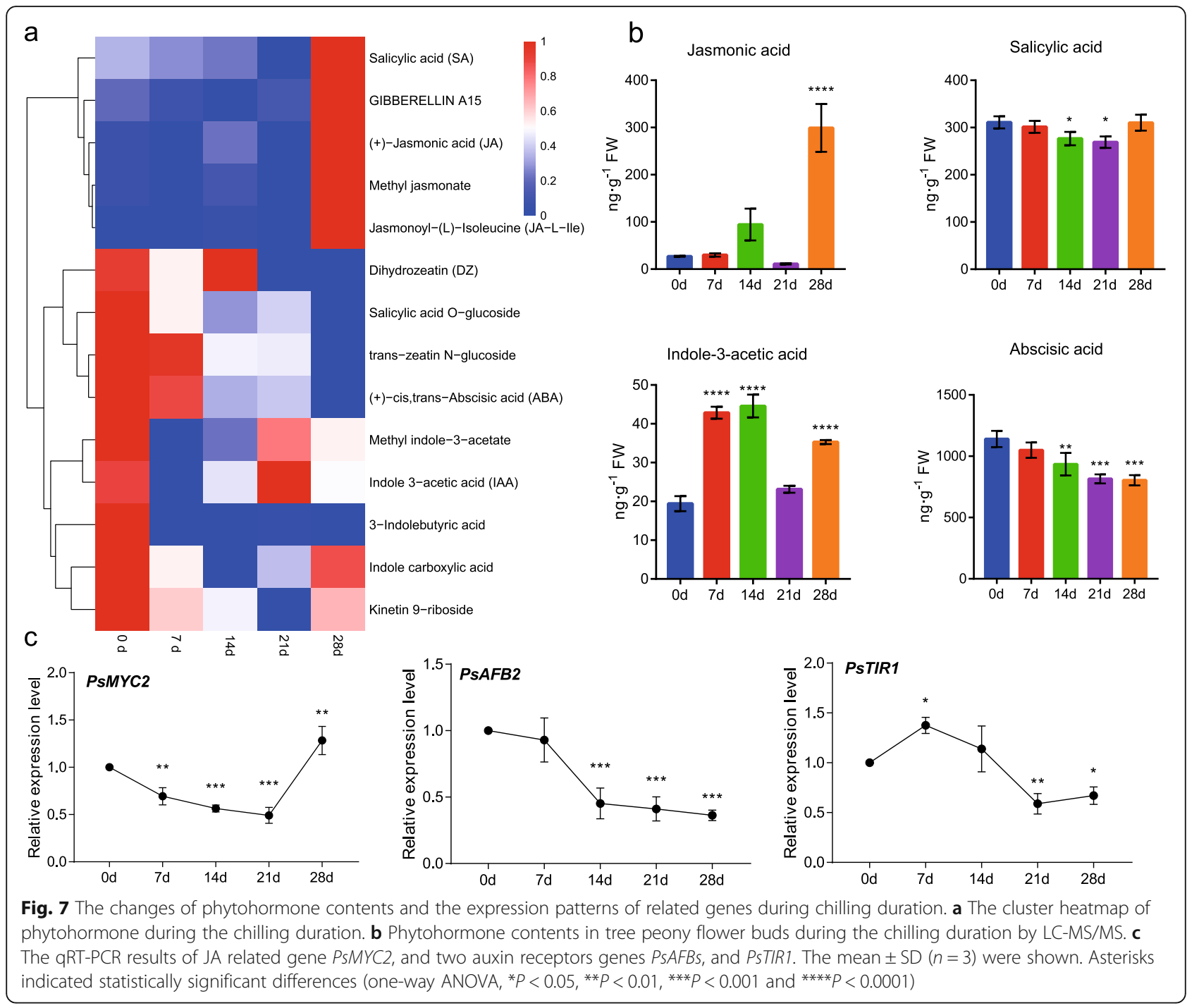

and carbohydrate metabolism [7, 13, 27]. Here, substance changes were detected by metabolomics analysis during chilling induced dormancy transition, and the expression of related genes were also analyzed by qRTPCR. Several metabolsim pathways were enriched and 118 metabolites were differential in the process..

\section{Starch degradation and EMP pathway were enhanced during dormancy release}

Carbohydrate is the basic energy substance of primary metabolism and secondary metabolism. Sugars metabolism and signal transduction involving in bud dormancy process had been revealed by RNA-seq [42]. Sugar metabolism genes (beta-amylase 5, alpha-amylase-like 1, sucrose synthase 3, and trehalose-phosphatase/synthase 7), sugar transporter genes (GT-2 like 1, sucrose-proton symporter 2, protein O-mannosyltransferase 5, and senescence-associated gene 29) and sugar signal transduction genes (glucose insensitive 2 and beta-fruct 4 ) had been proven to involve in dormancy release in poplar, grape and P. mume [42-44]. In this study, the contents of carbohydrates were continuously measured during the chilling induced dormancy transition of tree peony. Both starch and maltose showed downward trend before dormancy release, which were consistent with the increasing of amylase activity and sugar catabolism (Fig. 5b, d). At the same time, the enzyme activity of AMY gradually increased. Our recent results also revealed that the transcripts of PSAMY and PSBMY were up-regulated during the same process $[7,8,42]$. Together, these results indicated that chilling treatment promoted the degradation of starch during dormancy release in tree peony. After endodormancy release (21-28 d), the buds maintained active respiratory metabolism and amylase activity, but the contents of starch and maltose did not decrease significantly (Fig. 5b, d). It was speculated that a considerable amount of carbohydrates were transported from other parts to the buds, such as the root system (tree 
peony has a developed succulent root system), to meet the consumption of flower buds. In addition, the reopening of the material transport channel might be another important reason, which enabled the long-distance transport of carbohydrates [30].

In our study, starch was rapidly degraded at the beginning of the chilling duration due to high amylase activity, and sucrose was a kind of its intermediate product. The content of sucrose was the most abundant among the measured sugars, which implied that sucrose might also be used to transport of assimilates in tree peony rather than sorbitol as in apple because sucrose content was about 3200 folds higher than that of sorbitol. Sucrose was up-regulated till to $14 \mathrm{~d}$ and decreased afterward according to GC-MS/MS results (Fig. 5b), although sucrose synthase gene SUS1 was upregulated by chilling duration. Our results were different to that in Persian walnut with a persistent increase of sucrose [8]. Taken together, we speculated that sucrose mainly came from starch degradation, and were rapidly utilized for the following EMP pathway and so on. It was also found that sucrose accumulated, and sucrose synthase genes were up-regulated at the early stage of dormancy process in poplar and $P$. mume [42]. Therefore, sucrose might be used as an energy center to ensure the supply of glucose in dormancy transition of tree peony.

In addition, extensive degradation of starch should lead to glucose accumulation, but glucose and fructose were at a low level or down-regulated until the ecodormancy stage (Fig. 5a, b). Meanwhile, the contents of F6P and G6P increased significantly after chilling exposure, and thereafter decreased (Fig. 4 and 5a). Also, Our previous study showed that the transcripts $(H K$ and G6P) and enzyme activities of Hexokinase and glucose 6phosphate isomerase were also significantly up-regulated $[7,42]$. These results indicated that the EMP pathway was activated after chilling exposure in tree peony. Metabolomics results showed that dormancy release was an energy-consuming process. A large number of carbohydrates were broken down to produce enough substances and energy to promote dormancy release, which also providied a carbon chain for secondary metabolism.

\section{Flavonoids accumulation at ecodormancy stage}

As secondary metabolites, flavonoids play important roles in many processes of plant growth and development, such as color formation, stress resistance, etc. [15]. However, the changes and functions of flavonoids during dormancy transition were still poorly understood. Variations of flavonoids were analyzed during chilling duration process in our work. Flavonoids (e.g. quercetin, kaempferol, and apigenin) were synthesized in the early step of the flavonoid biosynthesis pathway, which were down-regulated in the whole endodormancy stage and sharply upregulated at $28 \mathrm{~d}$, corresponding to the decreasing expression of PsCHI (Fig. 6). The flavonoids accumulation at early stage of chilling treatment $(0 \mathrm{~d}$ and $7 \mathrm{~d}$ ) might be related to cold tolerance for winter survival. It was considered that the initial products of the flavonoids biosynthesis pathway, such as quercetin, kaempferol, apigenin, etc., inhibited the transport of polar auxin to regulate plant development [45]. Also, the correlation between pollen fertility and flavonoids have been found in maize and peanuts [23], and the silencing of chalcone synthase gene resultes in parthenocarpy in tomato [46]. Flavonols (in particular quercetin) is essential for pollen germination in tobacco [26]. In our results, flavonols showed higher levels at ecodormancy stage (Fig. 6a), when the flower buds were welldifferentiated, and stamens were clearly visible [34]. Therefore, it was speculated that flavonols might be involved in flower bud development at ecodormancy stage in tree peony.

In a recent study, Gu et al. (2019) suggested that anthocyanin accumulation occurred $10 \mathrm{~d}$ before anthesis in tree peony 'Qing Hai Hu Yin Bo' [47]. Here, we found anthocyanins were up-regulated after endodormancy release, accumulating in large amounts at ecodormancy stage in tree peony 'Luhehong' (Fig. 6). The results implied that the floral pigments might begin their synthesis and accumulation before the bud entering into endodormancy with a peak at ecodormancy period. Cyanidinbased glycosides such as Cy3Glu and Cy3Glu5Glu were the most abundant anthocyanins in the petal blotches of 35 cultivars [48], and they were also the most abundant anthocyanins in the buds of 'Luhehong' (Fig. 6b). We hypothesized that chilling-induced dormancy release synchronously activated anthocyanin synthesis and accumulation.

Taken together, flavonoids synthesis and accumulation could be actived by prolonged chilling, and dormancy release might accelerate flower bud development through flavonoids accumulation. To our knowledge, it was the first report to describe the changes and role of flavonoids during dormancy transition in perennial woody plants.

\section{The roles of phytohormone during dormancy transition}

In perennial woody plants, ABA and GA have been widely proven to regulate bud dormancy. Recent researches revealed the antagnism between ABA and GA in bud dormancy. The GA and ABA pathways are found to be the most enriched in the comparison of different dormancy stages in grape [49]. A MADS-box (DAM) family gene, SHORT VEGETATIVE PHASE-like (SVL) have been found to play a key role in ABA-mediated bud dormancy in poplar. In SVL RNAi strain, the expression of FT1 is significantly up-regulated, and GA 
biosynthesis key enzyme gene GA20ox is up-regulated to promote bud rupture [32]. Further research found that SVL could directly bind to the promoters of GA2ox8 and CALS1 to induce their expression, reduce the levels of active GAs and control the closure of plasmodesmata, thereby maintain dormancy status [50]. Chilling accumulation reduces the level of $A B A$, which in turn suppresses the expression of $S V L$ and promotes the biosynthesis of GAs, and finally break bud dormancy [36]. In our work, ABA was persistently down-regulated along with the chilling duration (Fig. 7), which was similar to grape during domancy transition process [51]. Gibberellin 15, the precursor of bioactive gibberellins, was up-regulated gradually (Fig. 7a). Meanwhile, the expression of PsGA200x was up-regulated, and the content of GAs increased with chilling accumulation in tree peony $[51,52]$. Therefore, there might be similar mechanisms between ABA and GA-regulated dormancy in poplar and tree peony.

Auxins and cytokinins (CKs) play antagonistic roles in meristems of many plants [53]. Previous researches had shown that CKs play a positive role in hydrogen cyanamide-induced bud dormancy release in grape [54], but that of IAA is still ambiguous until now. In our study, the level of CKs (dihydrozeatin, trans-zeatin $\mathrm{N}$ glucoside and kinetin 9-rboside) decreased after chilling exposure, and IAA was up-regulated by chilling through metabolomics and LC-MS/MS analysis (Fig. 7). TRAN SPORT INHIBITOR RESPONSE1/AUXIN SIGNALING F-BOX PROTEIN (TIR1/AFB) family are known as auxin receptors [55]. The transcripts of PSTIR1 were significantly induced and consistent with the IAA contents variation (Fig. 7c), which implied a positive role of IAA during dormancy release in tree peony. These results were different from the upregulation of CKs in P. kingianum and $P$. mume during the same process $[56,57]$. Therefore, the regulations of CKs and IAA in dormancy release were not a common mechanism in different perennial plants, and they might not be the key factors in dormancy regulation.

Usually, SA and JA are regarded as stress response hormones, rather than function on dormancy regulation [58, 59]. Recently, Ionescu et al. (2017) proposed that the upregulation of JA-Ile induced the expression of MYB21 (myeloblastosis viral oncogene homolog B21) and MYB108 to participate in the flower development process during $\mathrm{HC}$-induced dormancy release in sweet cherry [57]. In our results, JAs were dramatically upregulated when entering ecodormancy period, along with a transcriptional increase of $P S M Y C 2$, a key transcription factor for JA signal transduction (Fig. 7). The results indicated that JA signal transduction was activated after the fufilment of chilling accumulation. In a recent study, the mechanism of JA promoting the anthocyanins accumulation was revealed in Arabidopsis. When the JA content increases, the inhibitory effect of JAZs (jasmonate ZIM-domain) protein on MYB-bHLH-WD40 complex is released, which will promote the expression of $D F R$ and $A N S$, and the accumulation of anthocyanins [60]. Interestingly, PSDFR and PSANS also significantly increased, and anthocyanins presented higher levels at $28 \mathrm{~d}$ chilling treatment in our study (Fig. 7). It was hypothesized that JA additionally involved in anthocyanin accumulation after endodormancy release in tree peony. Additionally, SA involves in the response to lowtemperature stress, for SA and glucosyl SA accumulating after low-temperature exposure [58]. The use of exogenous SA improves the cold tolerance of corn, cucumber, and rice [59]. The accumulations of SA and Salicylic acid $\mathrm{O}$-glucoside were also observed during chilling induced dormancy release in tree peony (Fig. 7), it might be the response of buds to low-temperature stress.

\section{Conclusions}

In summary, we systematically revealed the metabolomic changes during the chilling induced dormancy transition of tree peony, and a total of 511 substances and 118 DMs were identified. Chilling accumulation promoted the degradation of starch and enhanced the activity of EMP, providing adequate energy and substances for secondary metabolism required by dormancy release and bud burst. Flavonoid was accumulated by sufficient chilling duration along with endodormancy release. Furthermore, we also reported phytohormone changes during the dormancy transition in tree peony. Prolonged chilling exposure declined ABA content, but promoted JA and GA accumulation at the end of dormancy. Taken together, we proposed a work model of dormancy transition induced by chilling according to the metabonomics analysis (Fig. 8). Our results might help to better understand the dormancy transition of perennial plants.

\section{Materials and methods Plant materials}

Four-years-old tree peony plants (Paeonia suffruticosa cv. Luhehong) were provided by Tree Peony Research Institute of Qingdao Agricultural University, Qingdao, China. The plants were treated with continuous artificial chilling $\left(0-4{ }^{\circ} \mathrm{C}\right.$ refrigeratory storage, dark $\left./ 24 \mathrm{~h}\right)$ from Nov 12, 2018 with the daily average temperature less than $10^{\circ} \mathrm{C}$ as described previously [7]. At $0,7,14,21$ and $28 \mathrm{~d}$ after refrigerating treatment, buds were picked and scales were removed in each time point, frozen in liquid nitrogen, and stored at $-80{ }^{\circ} \mathrm{C}$ until further analysis. The samples from each three plants were harvested and mixed in each treatment. Three replicates (3 plants/ replicate) per group were set. 


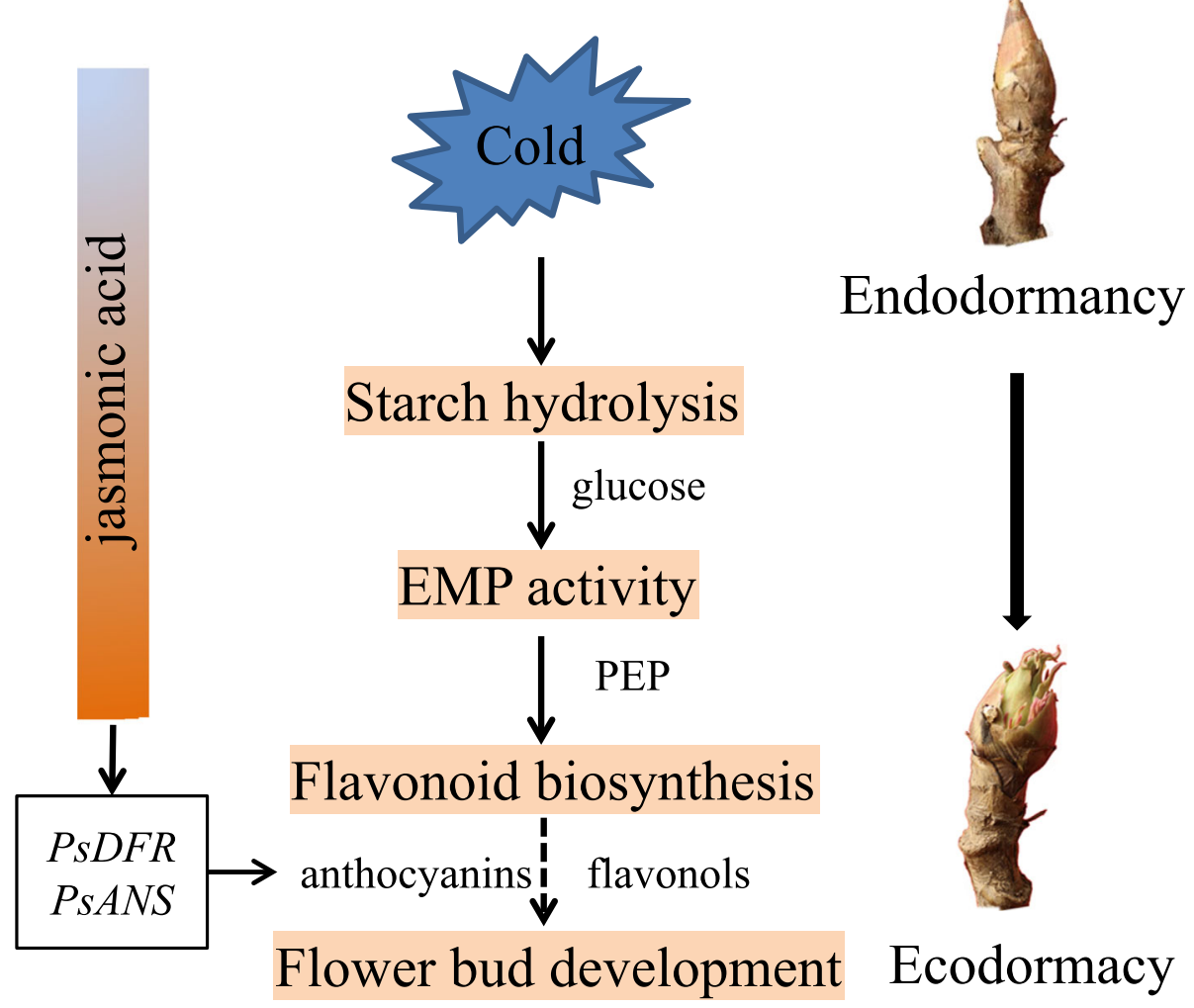

Fig. 8 Model of metabolite role during dormancy transition induced by chilling in tree peony. Starch degradation and EMP activation provide energy and material basis for flavonoid accumulation during endodormancy release. Flavonoid and anthocyanin accumulation might promote flower bud development at ecodormancy stage. Meanwhile, the accumulation of anthoyanin may be regulated by JA

\section{Sample preparation and extraction}

After freeze-drying, the samples were crushed at $30 \mathrm{~Hz}$ for 1.5 min using a mixing mill with zirconia beads (MM 400 , Retsch). Then powder (100 mg) was mixed with $70 \%$ methanol solution $(1 \mathrm{~mL}$, containing $0.1 \mathrm{mg} / \mathrm{L}$ lidocaine as an internal standard) at $4{ }^{\circ} \mathrm{C}$ overnight. After centrifugation $(10,000 \mathrm{~g}, 10 \mathrm{~min})$, the supernatant was filtered (scaa-104, aperture $0.22 \mu \mathrm{m}$; ANPEL, Shanghai, China) and analyzed by LC-MS/MS. Quality control (QC) samples were mixed with all samples to test the repeatability of the whole experiment [61].

\section{Liquid chromatography electrospray ionisation tandem mass spectrometry (LC-ESI-MS/MS)}

The extracted compounds were analyzed using an LCESI-MS/MS system (UPLC, Shim-pack UFLC SHIM ADZU CBM20A; MS/MS, Applied Biosystems 4500 QTRAP) by Gene Denovo Biotechnology Co. Ltd., Guangzhou, China [62]. Firstly, the samples $(5 \mu \mathrm{L})$ were added into a Waters ACQUITY UPLC HSS T3 $\mathrm{C}_{18}$ chromatographic column $(2.1 \mathrm{~mm} \times 100 \mathrm{~mm}, 1.8 \mathrm{~m})$ with a flow rate of $0.4 \mathrm{~mL} / \mathrm{min}$ at $40{ }^{\circ} \mathrm{C}$. The water and acetonitrile were acidificated with $0.04 \%$ acetic acid, which were used as the mobile phases of stages $A$ and B, respectively. The phase gradients to separate the compounds was as Chen et al. [62]. The Linear ion trap (LIT) and triple quadrupole (QQQ) scans were performed on the triple quadrupole linear ion TRAP mass spectrometer (QTRAP). Then AB Sciex QTRAP4500 (ABQ) system was equipped with an ESI-Turbo IonSpray interface. ABQ was ran in positive ion mode, and Analyst 1.6.1 software (AB Sciex) was used to operate according to Chen et al. [62].

\section{Qualitative and quantitative determination of metabolites} The qualitative analysis of metabolites was performed based on the public metabolite database (e.g. MassBank and KNApSAcK) and the METLIN database (MWDB) $[37,38]$. The repetitive signal (e.g. $\mathrm{K}^{+}, \mathrm{Na}^{+}, \mathrm{NH}_{4}{ }^{+}$, and other large molecular weight species) were removed during the analysis process. The metabolites were quantified by multiple reaction monitoring (MRM) of triple quadrupole mass spectrometry. The total ion chromatogram (TIC) and extracted ion chromatogram (EIC or Xic) of QC samples were derived to summarize the metabolite spectra of all samples and calculate the area of each peak. The MultiaQuant software (v 3.0.3) was used to integrate and calibrate the peaks. 
Principal component analysis (PCA) and orthogonal projection to latent structures-discriminant analysis (OPLS-DA)

To initially visualize the differences between the groups, the $\mathrm{R}$ package "ropls" was employed for principal component analysis (PCA) (http://bioconductor.org/packages/release/ bioc/html/ropls.html). Orthogonal projection to latent structures-discriminant analysis (OPLS-DA) is an development of PLS-DA, which comprises an Orthogonal Signal Correction (OSC) filter into a PLS model. The OPLS-DA model was used to analyze all comparison groups. And, subsequent model tests and differential metabolite screening were analyzed using OPLS-DA results.

\section{Differential metabolites analysis and KEGG analysis}

The most distinguishable metabolites between every two groups were ranked by the variable importance of the projection (VIP) score according to OPLS model. The threshold for VIP was set to 1.0. Besides, Student's t test was used as to screen differential metabolites. Those with $P<0.05$ and VIP $\geq 1$ were considered as differential metabolites between two groups. The KEGG Orthology software (http://kobas.cbi.pku.edu.cn/) was used for KEGG pathway analysis.

\section{RNA extraction and real time quantitative PCR analysis}

After $0,7,14,21$ and $28 \mathrm{~d} 0-4{ }^{\circ} \mathrm{C}$ treatment, the total RNA was isolated from $P$. suffruticosa flower buds according to the protocol of RNA isolation kit (TaKaRa, Dalian, China). The DNase I (TaKaRa, Dalian, China) was used to remove genomic DNA. The first strand cDNA was synthesized by HiScript III RT SuperMix for qPCR (+gDNA wiper) (Vazyme, Nanjing, China). qRTPCR was carried out using ChamQ Universal SYBR qPCR Master Mix (Vazyme, Nanjing, China) following the manufacturer's protocol. The detailed reaction system and procedure were similar to the previous study [36]. Additionally, the P. suffruticosa Actin was used as an internal control to normalize the transcriptional levels based on our previous results of different candidate control genes (Actin, beta-tubulin, alpha-tubulin and $\left.60 S-L_{11}\right)$ [63]. The Primer Premier 6 was used to designed specific primers (Table S4), and the candidate genes were selected based on our previous transcriptional profile [36], with their phylogenetic trees presented in Fig. S4. The relative expression levels were calculated using $2^{-\Delta \Delta C t}$ method [64].

\section{Measurements of sugar contents}

Three flower buds of every repeat at five chilling points were crushed by a crusher (MM 400, Retsch) containing zirconia beads at $30 \mathrm{~Hz}$. A total of $20 \mathrm{mg}$ powder was added into $500 \mu \mathrm{L}$ of methanol: isopropanol: water (3: 3: $2, \mathrm{~V} / \mathrm{V} / \mathrm{V})$ solution. The supernatant $(50 \mu \mathrm{L})$ were taken and evaporated in nitrogen after being vortexed ( $3 \mathrm{~min}$ ) and sonicated $(30 \mathrm{~min})$, with adding internal standard. After evaporated in a nitrogen stream and freeze-drying, the residue was further derivated as follows: Firstly, the small molecule carbohydrate was mixed with methoxine hydrochloride solution $(100 \mu \mathrm{L})$ in the $1.5 \mathrm{~mL}$ tubes. Secondly, bistrifluoroacetamide $(100 \mu \mathrm{L})$ was added to the solution at $37^{\circ} \mathrm{C}$ for $2 \mathrm{~h}$. After vortexed, the mixture was incubated at $37^{\circ} \mathrm{C}$ for $30 \mathrm{~min}$. N-Hexane was used as dilution fusion. Then, mixture was detected by MetWare (http://www.metware.cn/) based on the Agilent7890B7000D GC-MS/MS platform, and the parameters were set as reported by Gómez-González et al. [65] .

\section{Measurements of starch content and AMY enzyme activity}

The contents of starch at five chilling points was determined according to the previous method with little change, respectively [66]. The crushed tree peony buds $(0.1 \mathrm{~g})$ were extracted in $7.2 \mathrm{~mL}$ of ethanol $(80 \%)$ at $80^{\circ} \mathrm{C}$ for $30 \mathrm{~min}$. The extract was centrifuged for $30 \mathrm{~min}$, and the precipitate was gelatinized in a boiling water bath for $15 \mathrm{~min}$. Concentrated sulfuric acid was added to the precipitate to dehydrate monosaccharides into aldehyde compounds. The anthrone reagent was used to react with the test solution. The absorbance at $640 \mathrm{~nm}$ was recorded with a spectrophotometer (HITACHI, Japan).

The AMY enzyme activity was determined according to the method of Huggins and Russell (1948) with minor modifications [67]. About $0.2 \mathrm{~g}$ flower buds after different chilling fulfilling $(0,7,14,21$ and $28 \mathrm{~d})$ from tree peony was ground into a homogenate, and centrifuged to collect the supernatant, respectively. The supernatant was incubated at $70^{\circ} \mathrm{C}$ for $30 \mathrm{~min}$ to inactivate $\beta$ amylase. The samples were reacted with 3,5-dinitrosalicylic acid. The absorbance at $525 \mathrm{~nm}$ was recorded with a spectrophotometer (HITACHI, Japan).

\section{Measurements of hormone contents}

Approximately $2 \mathrm{~g}$ fresh weight buds per repeat were taken from different chilling treatments. The contents of phytohormones were determined by the Wuhan Greensword Creation Technology Company (http://www. greenswordcreation.com) based on LC-MS/MS analysis according to a previously reported method with minor modification [68].

\section{Statistical analysis}

Means and standard errors were calculated using Graphpad Prism 7 (San Diego, USA). Analysis of variance (One way ANOVA) was used to compare statistical differences and levels of gene expression between treatments and control. 


\section{Supplementary information}

Supplementary information accompanies this paper at https://doi.org/10. 1186/s12870-020-02692-x.

Additional file 1: Table S1 Metabolites identified by LC-ESI-MS/MS and annotated.

Additional file 2: Table S2. KEGG pathway enrichment of dormancy release related DMs.

Additional file 3: Table S3. DMs related to dormancy release, which were also involved in metabolic pathways.

Additional file 4: Table S4. Primer sequences used for qRT-PCR analysis in current study.

Additional file 5: Table S5. The accession number of protein used in phylogenetic analysis.

Additional file 6: Figure S1. OPLS-DA score plot of each comparison group.

Additional file 7: Figure S2. Venn diagram of 4 comparative metabolites. (a) up-regulated metabolites. (b) down-regulated metabolites.

Additional file 8: Figure S3. The KEGG enrichment analysis for top 20 of differential metabolites. Metabolites with a $P$ value of T test of $<0.05$ and VIP $\geq 1$ were identified as differential metabolites between each two treatments.

Additional file 9: Figure S4. Phylogenetic analysis of related-gene used for expression analysis in this study. The amino acid sequences of proteins were aligned with Clustal W, and phylogenetic trees were constructed in MEGA 7 using Neighbor-Joining method with the following options: partial deletion and replicate bootstrap (1000). The protein accession number were shown in Table S5. (a) Sucrose-related genes. (b) Flavonoid-related genes. (c) Phytohormone-related genes.

\section{Abbreviations}

QC: Quality control; OPLS-DA: Orthogonal projection to latent structuresdiscriminant analysis; DMs: Differential metabolites; SD: Standard deviation; VIP: Variable importance in the project; GC-MS/MS: Gas chromatographymass spectrometry; LC-MS/MS: Liquid chromatography electrospray ionisation tandem mass spectrometry; EBG: Early biosynthetic gene of flavonoid biosynthesis; LBG: Late biosynthetic gene of flavonoid biosynthesis; bHLH: Basic helix-loop-helix protein; WD40: WD repeat protein; ANOVA: Analysis of variance

\section{Acknowledgements}

The authors greatly acknowledge Qingyan Shu at the Institute of Botany, Chinese Academy of Sciences for providing useful supervision of flavonoids analysis.

\section{Authors' contributions}

GSP and ZYX conceived and designed the experimental plan. ZT, CXZ, ZY and LCY conducted the experiments. ZT and YYC wrote the manuscript. GSP and ZYX revised the manuscript. All authors read and approved the final manuscript.

\section{Funding}

This work was supported by the grants from the National Natural Science Foundation of China (31672194, 31872145 and 31972452) and National Key R\&D Program of China (2018YFD1000403). The funding bodies had no role in the design of the study, the collection, analysis, and interpretation of data, or in writing the manuscript.

\section{Availability of data and materials}

The datasets used and/or analysed during the current study available from the corresponding author on reasonable request. The sequences of the genes used in this study are available at Genbank (https://www.ncbi.nlm.nih. gov/genbank/), and the accession numbers are as follows: PSCHS, JN105300.1; PSCHI, ADK55061.1; PSF3H, HQ283447.1; PSDFR, HQ283448.1; PSANS, KJ466969.1; PS3GT, MT702582; PSAFB2, MT702583; PSTIR1, MT702589; PSMYC2, MT702586; PSCIN, MT702584; PSCWIN, MT702585; PSVIN, MT702590; PSSUS1, MT702587; PSSUS2, MT702588.
Ethics approval and consent to participate

Not applicable.

\section{Consent for publication}

Not applicable.

\section{Competing interests}

The authors declare that they have no competing interests.

\section{Author details}

${ }^{1}$ College of Life Sciences, Qingdao Agricultural University, Qingdao 266109, China. ${ }^{2}$ University Key Laboratory of Plant Biotechnology in Shandong Province, Qingdao 266109, China.

Received: 21 July 2020 Accepted: 8 October 2020

Published online: 23 October 2020

\section{References}

1. Anderson JV, Gesch RW, Jia Y, Chao WS, Horvath DP. Seasonal shifts in dormancy status, carbohydrate metabolism, and related gene expression in crown buds of leafy spurge. Plant Cell Environ. 2005;28(12):1567-78.

2. Lang GA, Early JD, Darnell RL, Martin GC. Endo-, Para- and ecodormancy: physiological terminology and classification for dormancy research. Hortscience. 1987;22:371-7.

3. Rohde A, Bhalerao RP. Plant dormancy in the perennial context. Trends Plant Sci. 2007;12(5):217-23.

4. Raghuram N. Knowing when to grow: signals regulating bud dormancy. Trends Plant Sci. 2003;9(1):9-12.

5. Saure MC. Dormancy release in deciduous fruit trees. Hortic Rev. 2011;7: 239-300.

6. Rhie $\mathrm{YH}$, Jung $\mathrm{HH}$, Kim KS. Chilling requirement for breaking dormancy and flowering in Paeonia lactiflora 'Taebaek' and 'Mulsurae'. Hortic Environ Biote. 2012;53(4):277-82

7. Zhang Y, Yu D, Liu C, Gai S. Dynamic of carbohydrate metabolism and the related genes highlights PPP pathway activation during chilling induced bud dormancy release in tree peony (Paeonia suffruticosa). Sci HorticAmsterdam. 2018;242:36-43.

8. Gholizadeh J, Sadeghipour HR, Abdolzadeh A, Hemmati K, Hassani D, Vahdati K. Redox rather than carbohydrate metabolism differentiates endodormant lateral buds in walnut cultivars with contrasting chilling requirements. Sci Hortic-Amsterdam. 2017;225:29-37.

9. Aslani Aslamarz A, Vahdati K, Rahemi M, Hasani D. Estimation of chilling and heat requirements of some Persian walnut cultivars and genotypes. HortScience. 2009;44(3):697-701.

10. Bonhomme M, Peuch M, Ameglio T, Rageau R, Guilliot A, Decourteix M, Alves G, Sakr S, Laconinte A. Carbohydrate uptake from xylem vessels and its distribution among stem tissues and buds in walnut (Juglans regia L.). Tree Physiol. 2009;30:89-102.

11. Gonzalez-Rossia D, Reig C, Dovis V, Gariglio N, Agusti M. Changes on carbohydrates and nitrogen content in the bark tissues induced by artifificial chilling and its relationship with dormancy bud break in Prunus sp. Sci Hortic-Amsterdam. 2008;118:275-81.

12. Takemura $Y$, Kuroki $K$, Jiang M, Matsumoto K. Identifification of the expressed protein and the impact of change in ascorbate peroxidase activity related to endodormancy breaking in Pyrus pyrifolia. Plant Physiol Biochem. 2015;86:121-9.

13. Zhang Y, Wang Y, Gao X, Liu C, Gai S. Identification and characterization of microRNAs in tree peony during chilling induced dormancy release by high-throughput sequencing. Sci Rep-UK. 2018;8(1):1-14.

14. Xue J, Tang Y, Wang S, Xue Y, Liu X, Zhang X. Evaluation of dry and wet storage on vase quality of cut peony based on the regulation of starch and sucrose metabolism. Postharvest Biol Tec. 2019;155:11-9.

15. Fallahi H, Scofield GN, Badger MR, Chow WS, Furbank RT, Ruan YL. Localization of sucrose synthase in developing seed and siliques of Arabidopsis thaliana reveals diverse roles for SUS during development. J Exp Bot. 2008;59(12):3283-95

16. Sturm A. Invertases. Primary structures, functions, and roles in plant development and sucrose partitioning. Plant Physiol. 1999;121(1):1-7.

17. Wan H, Wu L, Yang Y, Zhou G, Ruan Y. Evolution of sucrose metabolism: the dichotomy of invertases and beyond. Trends Plant Sci. 2018;23(2):163-77. 
18. Wang SY, Jiao HJ, Faust M. Changes in metabolic enzyme activities during thidiazuron-induced lateral budbreak of apple. Hortscience. 1991;26(2):171-3.

19. Halaly T, Pang X, Batikoff T, Crane O, Keren A, Venkateswari J, Ogrodovitch A, Sadka A, Lavee S, Or E. Similar mechanisms might be triggered by alternative external stimuli that induce dormancy release in grape buds. Planta. 2008;228(1):79-88.

20. Vergara R, Pérez FJ. Similarities between natural and chemically induced bud-endodormancy release in grapevine Vitis vinifera L. Sci HorticAmsterdam. 2010;125(4):648-53.

21. Shen C, Wang HB, Wang XD, Wang BL, Zheng XC, Shi XB, Liu WC, Liu FZ. Respiratory changes during dormancy of grape buds. Sci Agric Sin. 2013; 46(6):1201-7.

22. Mason MG, Ross JJ, Babst BA, Wienclaw BN, Beveridge CA. Sugar demand, not auxin, is the initial regulator of apical dominance. Proc Natl Acad Sci $U$ S A. 2014;111(16):6092-7.

23. Ferreyra ML, Rius SP, Casati P. Flavonoids: biosynthesis, biological functions, and biotechnological applications. Front Plant Sci. 2012;3:222

24. Xu W, Dubos C, Lepiniec L. Transcriptional control of flavonoid biosynthesis by MYB-bHLH-WDR complexes. Trends Plant Sci. 2015;20(3):176-85.

25. Pollastri S, Tattini M. Flavonols: old compounds for old roles. Ann BotLondon. 2011;7(108):1225-33.

26. Mahajan M, Ahuja PS, Yadav SK. Post-transcriptional silencing of flavonol synthase mRNA in tobacco leads to fruits with arrested seed set. PLoS One. 2011;6:e28315.

27. Sun C, Deng L, Du M, Zhao J, Chen Q, Huang T, Jiang H, Li C, Li C. A transcriptional network promotes anthocyanin biosynthesis in tomato flesh. Mol Plant. 2019;13(1):42-58.

28. Welling A, Palva ET. Molecular control of cold acclimation in trees. Physiol Plant. 2006;127(2):167-81.

29. Singh P, Dave A, Vaistij FE, Worrall D, Holroyd GH, Wells JG, Kaminski F, Graham IA, Roberts MR. Jasmonic acid-dependent regulation of seed dormancy following maternal herbivory in Arabidopsis. New Phytol. 2017; 214(4):1702-11.

30. Rinne PL, Welling A, Vahala J, Ripel L, Ruonala R, Kangasjärvi J, Schoot C. Chilling of dormant buds hyperinduces FLOWERING LOCUS T and recruits GA-inducible 1,3- $\beta$-glucanases to reopen signal conduits and release dormancy in Populus. Plant Cell. 2011;23(1):130-46.

31. Zheng C, Halaly T, Acheampong AK, Takebayashi Y, Jikumaru Y, Kamiya Y, Etti YO. Abscisic acid (ABA) regulates grape bud dormancy, and dormancy release stimuli may act through modification of $A B A$ metabolism. J Exp Bot. 2015;66(5):1527-42.

32. Singh RK, Maurya JP, Azeez A, Miskolczi P, Tylewicz S, Stojkovič K, Delhomme N, Busov V, Bhalerao RP. A genetic network mediating the control of bud break in hybrid aspen. Nat Commun. 2018;9(1):4173.

33. Busov VB. Plant development: dual roles of poplar SVL in vegetative bud dormancy. Curr Biot. 2019;29(2):R68-70.

34. Xin H, Zhang Y, Wang X, Liu C, Feng W, Gai S. Morphological, anatomical and DNA methylation changes of tree peony buds during chilling induced dormancy release. Plant Physiol Bioch. 2019;144:64-72.

35. Huang $X$, Zhu W, Dai S, Gai S, Zheng G, Zheng C. The involvement of mitochondrial phosphate transporter in accelerating bud dormancy release during chilling treatment of tree peony (Paeonia suffruticosa). Planta. 2008; 228(4):545-52

36. Gai S, Zhang Y, Liu C, Zhang Y, Zheng G. Transcript profiling of Paoenia ostii during artificial chilling induced dormancy release identifies activation of GA pathway and carbohydrate metabolism. PLoS One. 2013;8:e55297.

37. Wishart DS, Jewison T, Guo AC, Wilson M, Knox C, Liu Y, Djoumbou Y, Mandal R, Aziat F, Dong E, et al. HMDB 3.0-the human Metabolome database in 2013. Nucleic Acids Res. 2012;41(D1):D801-7.

38. Zhu Z, Schultz AW, Wang J, Johnson CH, Yannone SM, Patti GJ, Siuzdak G. Liquid chromatography quadrupole time-of-flight mass spectrometry characterization of metabolites guided by the METLIN database. Nat Protoc. 2013;8(3):451-60.

39. Breeze E. Master MYCs: MYC2, the Jasmonate signaling "master switch". Plant Cell. 2019;31(1):9-10.

40. Maurya JP, Bhalerao RP. Photoperiod- and temperature-mediated control of growth cessation and dormancy in trees: a molecular perspective. Ann BotLondon. 2017;120(3):351-60.

41. Vahdati K, Aslamarz AA, Rahemi M, Hassani D, Leslie C. Mechanism of seed dormancy and its relationship to bud dormancy in Persian walnut. Environ Exp Bot. 2012;75:74-82.
42. Zhang Z, Zhuo X, Zhao K, Zheng T, Han Y, Yuan C, Zhang Q. Transcriptome profiles reveal the crucial roles of hormone and sugar in the bud dormancy of Prunus mume. Sci Rep-UK. 2018;8(1):5090.

43. Ruttink T, Arend M, Morreel K, Storme V, Rombauts S, Fromm J, Bhalerao RP, Boerjan W, Rohde A. A molecular timetable for apical bud formation and dormancy induction in poplar. Plant Cell. 2007;19(8):2370-90.

44. Díaz-Riquelme J, Grimplet J, Martínez-Zapater JM, Carmona MJ. Transcriptome variation along bud development in grapevine (Vitis vinifera L.). BMC Plant Biol. 2012;12(1):181.

45. Lewis DR, Ramirez MV, Miller ND, Vallabhaneni P, Ray WK, Helm RF, Winkel BSJ, Muday GK. Auxin and ethylene induce flavonol accumulation through distinct transcriptional networks. Plant Physiol. 2011;156(1):144-64.

46. Schijlen EG, Vos CH, Martens S, Jonker HH, Rosin FM, Molthoff JW, Tikunov YM, Angenent GC, Tunen AJ, Bovy AG. RNA interference silencing of chalcone synthase, the first step in the flavonoid biosynthesis pathway, leads to parthenocarpic tomato fruits. Plant Physiol. 2007;144(3):1520-30.

47. Gu Z, Zhu J, Hao Q, Yuan Y, Duan Y, Men S, Wang Q, Hou Q, Liu Z, Shu Q, et al. A novel R2R3-MYB transcription factor contributes to petal blotch formation by regulating organ-specific expression of PsCHS in tree peony (Paeonia suffruticosa). Plant Cell Physiol. 2019;60(3):599-611.

48. Zhang J, Wang L, Shu Q, Liu ZA, Li C, Zhang J, Wei X, Tian D. Comparison of anthocyanins in non-blotches and blotches of the petals of Xibei tree peony. Sci Hortic-Amsterdam. 2007;2(114):104-11.

49. Singh RK, Miskolczi P, Maurya JP, Bhalerao RP. A tree ortholog of SHORT VEGETATIVE PHASE floral repressor mediates photoperiodic control of bud dormancy. Curr Biot. 2019;29(1):128-33.

50. Khalil-Ur-Rehman M, Sun L, Li CX, Faheem M, Wang W, Tao JM. Comparative RNA-seq based transcriptomic analysis of bud dormancy in grape. BMC Plant Biol. 2017;17(1):18.

51. Zheng G, Gai S, Gai W. Changes of endogenous hormones during dormancy release by chilling in tree peony. Scientia Silvae Sinicae. 2009;2: $48-52$.

52. Zhang Y, Zhang W, Li Y, Liu C, Zheng G, Gai S. The study of PSGA20ox gene participating in Endo-dormancy release of flower buds by chilling treatment in tree peony. Acta Agriculturae Boreali-Sinica. 2014;3:22-6.

53. Moubayidin L, Mambro RD, Sabatini S. Cytokinin-auxin crosstalk. Trends Plant Sci. 2009;14(10):557-62.

54. Lombard PJ, Cook NC, Bellstedt DU. Endogenous cytokinin levels of table grape vines during spring budburst as influenced by hydrogen cyanamide application and pruning. Sci Hortic-Amsterdam. 2006;109(1):92-6.

55. Dahlke RI, Fraas S, Ullrich KK, Heinemann K, Romeiks M, Rickmeyer T, Klebe G, Palme K, Luethen H, Steffens B. Protoplast swelling and hypocotyl growth depend on different auxin signaling pathways. Plant Physiol. 2017; 175(2):982-94

56. Wang Y, Liu X, Su H, Yin S, Han C, Hao D, Dong X. The regulatory mechanism of chilling-induced dormancy transition from endo-dormancy to non-dormancy in Polygonatum kingianum coll.Et Hemsl rhizome bud. Plant Mol Biol. 2019;99(3):205-17.

57. Ionescu IA, López-Ortega G, Burow M, Bayo-Canha A, Junge A, Gericke O, Møller BL, Sánchez-Pérez R. Transcriptome and metabolite changes during hydrogen Cyanamide-induced floral bud break in sweet cherry. Front Plant Sci. 2017:8:1233.

58. Kosova K, Prasil IT, Vitamvas P, Dobrev P, Motyka V, Flokova K, Novak O, Tureckova V, Rolcik J, Pesek B, et al. Complex phytohormone responses during the cold acclimation of two wheat cultivars differing in cold tolerance, winter Samanta and spring Sandra. J Plant Physiol. 2012;169(6): 567-76.

59. Kang HM, Saltveit ME. Chilling tolerance of maize, cucumber and rice seedling leaves and roots are differentially affected by salicylic acid. Physiol Plant. 2002;115(4):571-6.

60. Li C, Shi L, Wang Y, Li W, Chen B, Zhu L, Fu Y. Arabidopsis ECAP is a new adaptor protein that connects JAZ repressors with the TPR2 co-repressor to suppress jasmonate-responsive anthocyanin accumulation. Mol Plant. 2020; 13(2):246-65

61. Wang A, Li R, Ren L, Gao X, Zhang Y, Ma Z, Ma D, Luo Y. A comparative metabolomics study of flavonoids in sweet potato with different flesh colors (Ipomoea batatas (L.) lam). Food Chem. 2018;260:124-34.

62. Chen W, Gong L, Guo Z, Wang W, Zhang H, Liu X, Yu S, Aaron G. A novel integrated method for large-scale detection, identification, and quantification of widely targeted metabolites: application in the study of rice metabolomics. Mol Plant. 2013;6(6):1769-80. 
63. Zhang YX, Gai SP, Liu CY, Mu P, Zheng GS. Selection of control genes in real-time $\mathrm{gPCR}$ analysis during bud dormancy release in tree peony (Paeonia suffruticosa). Molecular Plant Breeding. 2011;9:1052-6.

64. Livak KJ, Schmittgen TD. Analysis of relative gene expression data using realtime quantitative $P C R$ and the $2^{-\Delta \Delta C T}$ method. Methods. 2001;25(4):402-8.

65. Gómez-González S, Ruiz-Jiménez J, Priego-Capote F, Luque De Castro MD. Qualitative and quantitative sugar profiling in olive fruits, leaves, and stems by gas chromatography-tandem mass spectrometry (GC-MS/MS) after ultrasound-assisted leaching. J Agr Food Chem. 2010;58(23):12292-9.

66. Hansen J, Møller I. Percolation of starch and soluble carbohydrates from plant tissue for quantitative determination with anthrone. Anal Biochem. 1975;68(1):87-94.

67. Huggins C, Russell PS. Colorimetric determination of amylase. Ann Surg. 1948;128(4):668-78.

68. Chen M, He X, Xia L, Ye T, Hou S, Huang Y, Yuan B, Wu Y, Feng Y. Highly sensitive and quantitative profiling of acidic phytohormones using derivatization approach coupled with nano-LC-ESI-Q-TOF-MS analysis. J Chromatogr B. 2012;905:67-74.

\section{Publisher's Note}

Springer Nature remains neutral with regard to jurisdictional claims in published maps and institutional affiliations.

Ready to submit your research? Choose BMC and benefit from:

- fast, convenient online submission

- thorough peer review by experienced researchers in your field

- rapid publication on acceptance

- support for research data, including large and complex data types

- gold Open Access which fosters wider collaboration and increased citations

- maximum visibility for your research: over $100 \mathrm{M}$ website views per year

At BMC, research is always in progress.

Learn more biomedcentral.com/submissions 